\title{
Construction of sentences with specific interpretability properties
}

\author{
by
}

Alan Stern (Cambridge, Mass.)

\begin{abstract}
A construction is presented for generating sentences that satisfy a recursively enumerable set of interpretability properties. This construction is then used to prove three previously announced results concerning the lattice of local interpretability types of theories (also known as the Lattice of Chapters).
\end{abstract}

The purpose of this paper is to prove three theorems originally announced without proof in [MPS]. The theorems are presented as Corollaries 10, 11, and 12 below; they state that no interval in the Lattice of Chapters bounded by r.e. theories is complemented, that the chapter of any r.e. theory is the meet of the chapters of finitely axiomatizable theories above it, and that the meet of the chapters of an r.e. set of finitely axiomatizable theories that all lie strictly above a given r.e. chapter is itself strictly above the given chapter. (As an interesting consequence of the second result it follows that there is a finitely axiomatizable theory $T$ such that $|\mathrm{ZF}|<|T|<|\mathrm{GB}|$, and as a consequence of the third it follows that for any r.e. set $A$ of sentences none of which has a finite model, there is a sentence $\tau$, also having no finite models, interpretable in every member of $A$.) The proofs of the corollaries are all based on a general technique for constructing sentences having very specific interpretability strengths. This general technique is given in Theorem 4, which is the main technical result of the paper.

Although this paper can be read independently of [MPS], the reader is encouraged to consult that work for further information and background on the Lattice of Chapters. For present purposes, the following remarks (up through Lemma 3) will serve as a brief introduction.

The collection of all first-order sentences can be partially ordered by the relation of interpretability. This yields a naturally induced equivalence relation: two sentences are equivalent if each is interpretable in the other. The equivalence classes are called chapters; the chapter containing a sentence 
$\alpha$ is written as $|\alpha|$. The chapters are ordered by interpretability of their constituents, and this ordering forms a lattice. A sentence having a finite model is interpretable by any sentence; the chapter consisting of all such sentences is the minimum element of the lattice. Similarly, any sentence is interpretable by an inconsistent sentence, so the chapter containing all inconsistent sentences is the maximum element of the lattice.

The lattice described here is only a sublattice of LC (the Lattice of Chapters). The full $\mathbf{L C}$ is a distributive algebraic lattice; it has chapters containing every first-order theory, not just the finitely axiomatizable ones. However, the results below concern individual sentences or, at most, recursively enumerable theories. The additional chapters in $\mathbf{L C}$ can be ignored.

The basic operations in $\mathbf{L C}$ are characterized by the next lemma.

LEMMA 1 (Mycielski). For any sentences $\alpha$ and $\beta$, the meet of $|\alpha|$ and $|\beta|$ in $\mathbf{L C}$ is equal to $|\alpha \vee \beta|$. If $\alpha$ and $\beta$ have no non-logical symbols in common (not even equality), then the join of $|\alpha|$ and $|\beta|$ in $\mathbf{L C}$ is equal to $|\alpha \wedge \beta|$.

The proof of Theorem 4 below will use a formalization of various syntactic and semantic notions, in a manner inspired by Vaught [V]. To simplify the exposition, only a minimal language will be treated.

Definition 2. The formula $\phi$ is called an $R$-formula if $\phi$ is built using only the binary relation symbol $R$ and the logical connectives $\neg$, $\vee$, and $\exists$. If $\phi$ also happens to be a sentence, it is called an $R$-sentence.

LEMMA 3 (Krajíček). For any sentence $\alpha$ there is an $R$-sentence $\alpha^{\prime}$ in the same chapter.

This lemma shows that for purposes of studying LC, it suffices to concentrate on $R$-sentences.

Some elementary concepts from lattice theory will be used. Recall that a subset $I$ of a lattice is called an ideal if $I$ is closed downward and under joins, i.e., if $a, b \in I$ and $c \leq(a \vee b)$ imply that $c \in I$. Dually, $F$ is called a filter if it is closed upward and under meets: $a, b \in F$ and $c \geq(a \wedge b)$ imply $c \in F$. Note that because interpretability is an r.e. notion, for any sentence $a$ the filter generated by $a$ (i.e., the set of sentences that can interpret $a$ ) is r.e. Similarly, for any r.e. theory $T$, the ideal generated by $T$ (the set of sentences interpretable in $T$ ) is also r.e.

The next theorem refers to indexed collections of sentences: an indexed collection is just a binary relation $I$ on sentences. For each index sentence $a$, the set $I_{a}$ is $\{b \mid(a, b) \in I\}$. An indexed collection of sentences is r.e. if it is r.e. as a binary relation. 
THEOREM 4. Let I and $F$ be r.e. indexed collections of sentences such that for any sentences $\alpha$ and $\beta$, if $\beta$ has a finite model then $\beta \in I_{\alpha}$ and if $\beta$ is inconsistent then $\beta \in F_{\alpha}$. Also suppose that for each $\alpha, \alpha \notin I_{\alpha} \cap F_{\alpha}$. Then the following are true.

(1) There is a sentence $\alpha$ such that $\alpha \notin I_{\alpha} \cup F_{\alpha}$.

(2) If every $I_{\alpha}$ is an ideal, then there is a sentence $\alpha$ such that $\alpha \notin F_{\alpha}$ and for every $\beta \in I_{\alpha},|\beta|<|\alpha|$ ( $\beta$ is interpretable in $\alpha$ but not vice versa).

(3) Similarly, if every $F_{\alpha}$ is a filter, then there is a sentence $\alpha$ such that $\alpha \notin I_{\alpha}$ and $|\alpha|<|\beta|$ for every $\beta \in F_{\alpha}$.

(4) If for every $\alpha, I_{\alpha}$ is an ideal, $F_{\alpha}$ is a filter, and in addition $\beta \in I_{\alpha}$, $\gamma \in F_{\alpha}$ imply $|\beta| \leq|\gamma|$, then there is a sentence $\alpha$ such that for all $\beta \in I_{\alpha}$ and $\gamma \in F_{\alpha},|\beta|<|\alpha|<|\gamma|$.

In most of the applications of this theorem, the sets $I_{\alpha}$ and $F_{\alpha}$ actually will not vary with the index $\alpha$ but will simply be some fixed pair. However, in at least one case the full generality is needed.

P r o of. The construction of the required sentences involves several steps, beginning with the introduction of Trachtenbrot's arithmetic, TA. (In fact, Trachtenbrot used TA in the proof of his theorem that the set of finitely satisfiable sentences is recursively inseparable from the set of inconsistent sentences (see $[\mathrm{T}]$ ). Part (1) above directly implies that result.) TA is a finitely axiomatizable theory of arithmetic much like Robinson's Q. However, it expresses the notions of successor, sum, and product by relations rather than by functions, because its intended models are initial segments of the natural numbers.

Definition 5. The language of TA contains the constant 0, binary relations $\leq$ and Succ, and ternary relations Sum and Prod. Its axioms are as follows:

$\leq$ is a discrete linear ordering, 0 is the least element, and there may or may not be a greatest element.

$\operatorname{Succ}(x, y)$ holds iff the successor of $x$ under $\leq$ is $y$.

Sum and Prod represent partial functions:

$$
\begin{aligned}
\operatorname{Sum}(x, y, z) & \wedge \operatorname{Sum}\left(x, y, z^{\prime}\right) \rightarrow z=z^{\prime}, \\
\operatorname{Prod}(x, y, z) & \wedge \operatorname{Prod}\left(x, y, z^{\prime}\right) \rightarrow z=z^{\prime} .
\end{aligned}
$$

The inductive definitions of Sum and Prod:

$$
\begin{gathered}
\operatorname{Sum}(x, 0, x), \\
\operatorname{Sum}(x, y, z) \wedge \operatorname{Succ}\left(y, y^{\prime}\right) \wedge \operatorname{Succ}\left(z, z^{\prime}\right) \rightarrow \operatorname{Sum}\left(x, y^{\prime}, z^{\prime}\right), \\
\operatorname{Prod}(x, 0,0), \\
\operatorname{Prod}(x, y, z) \wedge \operatorname{Succ}\left(y, y^{\prime}\right) \wedge \operatorname{Sum}\left(z, x, z^{\prime}\right) \rightarrow \operatorname{Prod}\left(x, y^{\prime}, z^{\prime}\right) .
\end{gathered}
$$


Lastly, two axioms that are needed to make TA categorical in all finite powers:

$$
\begin{aligned}
& \operatorname{Sum}\left(x, y^{\prime}, z^{\prime}\right) \wedge \operatorname{Succ}\left(y, y^{\prime}\right) \rightarrow \exists z \leq z^{\prime}\left(\operatorname{Succ}\left(z, z^{\prime}\right) \wedge \operatorname{Sum}(x, y, z)\right), \\
& \operatorname{Prod}\left(x, y^{\prime}, z^{\prime}\right) \wedge \operatorname{Succ}\left(y, y^{\prime}\right) \rightarrow \exists z \leq z^{\prime}\left(\operatorname{Sum}\left(z, x, z^{\prime}\right) \wedge \operatorname{Prod}(x, y, z)\right) .
\end{aligned}
$$

Certain facts about TA can be verified readily. For instance, every infinite model of TA contains an initial segment isomorphic to the natural numbers, while every finite model is isomorphic to an initial segment of the natural numbers. Unfortunately, because of these finite models a certain amount of care is needed in discussing theories based on TA. For any number $n$, let " $n$ exists" denote the sentence

$$
\exists y_{0}, \ldots, y_{n}\left(y_{0}=0 \wedge \operatorname{Succ}\left(y_{0}, y_{1}\right) \wedge \cdots \wedge \operatorname{Succ}\left(y_{n-1}, y_{n}\right)\right) .
$$

Then for any bounded formula $\psi\left(x_{1}, \ldots, x_{k}\right)$ and numbers $n_{1} \leq n, \ldots$ $\ldots, n_{k} \leq n$, it is clear that if $\psi\left(n_{1}, \ldots, n_{k}\right)$ is true then TA $\wedge$ " $n$ exists" $\vdash$ $\psi\left(n_{1}, \ldots, n_{k}\right)$.

The arithmetization of syntax can be carried out as usual in TA. The Gödel number of the formula $\phi$ will be written as $\# \phi$, and $\phi^{n}$ will stand for the formula whose Gödel number is $n$. For the most part the details do not matter, but for simple handling of $R$-formulas the Gödel numbering must obey some restrictions. In particular, if $\phi_{1}$ is a subformula of $\phi_{2}$, then $\# \phi_{1}$ must be $\leq \# \phi_{2}$. Also, the following relations must be expressible by bounded formulas of TA:

$$
\begin{array}{ll}
\operatorname{Ratom}(i, j, k) & \text { meaning that } \phi^{i} \text { is the atomic formula } R\left(x_{j}, x_{k}\right), \\
\operatorname{Neg}(i, j) & \text { meaning that } \phi^{i} \text { is } \neg \phi^{j}, \\
\operatorname{Disj}(i, j, k) & \text { meaning that } \phi^{i} \text { is } \phi^{j} \vee \phi^{k}, \text { and } \\
\operatorname{Exist}(i, j, k) & \text { meaning that } \phi^{i} \text { is } \exists x_{j} \phi^{k} .
\end{array}
$$

Of course, the Fixed-Point Lemma is crucial to the argument. For TA, the lemma takes the following form.

Lemma 6 (Gödel, Trachtenbrot). Let $\theta(x)$ be a formula (possibly in a larger language than that of $\mathrm{TA})$. Then there is a sentence $\alpha$ and $a$ bound $B \geq \# \alpha$ such that

$$
\mathrm{TA} \vdash \alpha \leftrightarrow \text { "B exists" } \wedge \theta(\# \alpha) .
$$

Proof. As in the usual case, but with slight adjustments for the peculiarities of TA. The $S_{m}^{n}$ Theorem states that the function $S_{1}^{1}$ which takes $\# \phi(x)$ and $n$ to $\# \phi(n)$ is recursive. Hence there is a bounded formula $S(x, y, z, w)$ of TA such that on the one hand, if $z=\# \phi(n)$ then $\exists w \geq z$ $S(\# \phi, n, z, w)$ is true, and on the other hand, if $z \neq \# \phi(n)$ then TA $\vdash$ 
$\forall w \neg S(\# \phi, n, z, w)$. Now let $U(x)$ be the generating formula

$$
\exists w \exists z \leq w(S(x, x, z, w) \wedge \theta(z)),
$$

and take $\alpha$ to be $U(\# U)$. With the bound $B$ chosen to be the least number $\geq \# \alpha$ such that $S(\# U, \# U, \# \alpha, B)$ is true, the lemma's conclusion is easy to check.

On with the proof of Theorem 4 . The sentences $\alpha$ referred to in the four parts of the theorem will all share a common portion, called $\Gamma$, that has been designed to facilitate the expression of certain syntactic and semantic notions. The language of $\Gamma$ consists of the language of TA; predicates $N$, $M, V$, Rfmla, and True; a binary function Var; and ternary relations $Q$ and Sat. The precise statement is given in Definition 7 below, but first here is an explanation of what $\Gamma$ means.

In models of $\Gamma$, the universe is partitioned by $N, M$, and $V$. The $N$ part is a model of TA, the $M$ part is an arbitrary set, and the $V$ part consists of elements representing sequences of members of $M$, indexed by members of $N$. These sequences are specified by Var: for $v \in V$ and $n \in N$, the $n$th element of the $v$ sequence is given by $\operatorname{Var}(v, n) . M$ serves as the universe for an indexed collection of binary relations $Q$ : for $n \in N$, the $n$th relation is given by $Q(n, x, y)$, also written as $Q_{n}(x, y)$. Naturally, Sat expresses the satisfaction relation on $M$ : for $n \in N, v \in V$, and any $R$-formula $\phi$, $\operatorname{Sat}(n, \# \phi, v)$ (also written as $\operatorname{Sat}_{n}(\# \phi, v)$ ) states that the variable assignment $v$ satisfies $\phi$, using $Q_{n}$ as the $R$ relation. The Rfmla predicate indicates which members of $N$ are Gödel numbers of $R$-formulas, and True indicates which $R$-sentences are true.

Definition 7. $\quad \Gamma$ is the conjunction of statements (1)-(7):

(1) The universe $=N \dot{\cup} M \dot{U} V$.

(2) The axioms of TA, relativized to $N$.

(3) $\forall v \in V \forall n \in N(\operatorname{Var}(v, n) \in M)$.

(4) $\forall v \in V \forall n \in N \forall x \in M \exists w \in V(\operatorname{SUB}(v, n, w) \wedge \operatorname{Var}(w, n)=x)$, where $\operatorname{SUB}(v, n, w)$ is an abbreviation for the formula $\forall m \in N(m \neq n \rightarrow$ $\operatorname{Var}(v, m)=\operatorname{Var}(w, m))$.

(5) The axiom

$$
\begin{aligned}
\operatorname{Rfmla}(f) \leftrightarrow f \in N \wedge(\exists g, h \leq & f)[\operatorname{Ratom}(f, g, h) \\
& \vee(\operatorname{Neg}(f, g) \wedge \operatorname{Rfmla}(g)) \\
& \vee(\operatorname{Disj}(f, g, h) \wedge \operatorname{Rfmla}(g) \wedge \operatorname{Rfmla}(h)) \\
& \vee(\operatorname{Exist}(f, g, h) \wedge \operatorname{Rfmla}(h))],
\end{aligned}
$$

where Ratom, Neg, Disj, and Exist are the bounded formulas mentioned earlier. 
(6) The axiom

$$
\begin{aligned}
\operatorname{Sat}_{n}(f, v) \leftrightarrow & \operatorname{Rfmla}(f) \wedge n \in N \wedge v \in V \wedge \\
\exists & , h \leq f\left[\left(\operatorname{Ratom}(f, g, h) \wedge Q_{n}(\operatorname{Var}(v, g), \operatorname{Var}(v, h))\right)\right. \\
& \vee\left(\operatorname{Neg}(f, g) \wedge \neg \operatorname{Sat}_{n}(g, v)\right) \\
& \left.\vee\left(\operatorname{Disj}(f, g, h) \wedge \operatorname{Sat}_{n}(g, v) \vee \operatorname{Sat}_{n}(h, v)\right)\right) \\
& \left.\vee\left(\operatorname{Exist}(f, g, h) \wedge \exists w \in V\left(\operatorname{SUB}(v, g, w) \wedge \operatorname{Sat}_{n}(h, w)\right)\right)\right],
\end{aligned}
$$

where SUB is as in (4) above.

(7) $\operatorname{True}(f) \leftrightarrow \operatorname{Rfmla}(f) \wedge \forall v \in V \operatorname{Sat}_{f}(f, v)$.

Note that in (7), the subscript in $\operatorname{Sat}_{f}(f, v)$ is $f$ itself. This effectively treats $R$-sentences as though they are all in disjoint languages, since for each $\phi$, True $(\# \phi)$ depends only on the structure with universe $M$ and the relation $Q_{\# \phi}$.

The next two lemmas express the key properties that $\Gamma$ has with regard to interpretability.

LEMMA 8. Let $J=\left\{j_{1}, \ldots, j_{k}\right\}$ be a finite set of Gödel numbers of $R$ sentences. Let $B$ be any upper bound for $J$, let $\Gamma^{J}$ be the sentence

$$
\Gamma \wedge \text { "B exists" } \wedge \operatorname{True}\left(j_{1}\right) \wedge \cdots \wedge \operatorname{True}\left(j_{k}\right),
$$

and let $C$ be the join of the chapters $\left|\phi^{j_{1}}\right|, \ldots,\left|\phi^{j_{k}}\right|$. Then $C \geq\left|\Gamma^{J}\right|$.

Note that as a limiting case of this lemma, when $J$ is empty the sentence $\Gamma^{J}=(\Gamma \wedge$ "B exists" $)$ has a finite model.

Lemma 9. Let $J$ be as above, let $\Gamma^{J}$ be the sentence

$$
\Gamma \wedge\left[\left(" j_{1} \text { exists" } \wedge \operatorname{True}\left(j_{1}\right)\right) \vee \cdots \vee\left(" j_{k} \text { exists" } \wedge \operatorname{True}\left(j_{k}\right)\right)\right],
$$

and let $C$ be the meet of the chapters $\left|\phi^{j_{1}}\right|, \ldots,\left|\phi^{j_{k}}\right|$. Then $C \leq\left|\Gamma^{J}\right|$.

Proof of Lemma 8 . Let $\bar{\phi}^{j}$ be the result of replacing the occurrences of $R$ in $\phi^{j}$ with a new relation symbol $R_{j}$. Then the sentences $\bar{\phi}^{j}(j \in J)$ are all in disjoint languages, so by Lemma 1 the sentence

$$
\phi^{J}=\bar{\phi}^{j_{1}} \wedge \cdots \wedge \bar{\phi}^{j_{k}}
$$

belongs to the chapter $C$. Hence it will suffice to find an interpretation $I$ of $\Gamma^{J}$ in $\phi^{J}$.

Here is a recipe for cooking up $I$. The interpretation $N^{I}$ of $N$ will just be the set $\{0, \ldots, B\}$. Since this is a finite set it is certainly definable in $\phi^{J}$, along with all the relations of TA on this set. $M^{I}$ will simply be the universe of $\phi^{J}$, while $V^{I}$ will be the $(B+1)$-power of the universe. The function $\operatorname{Var}^{I}$ can be defined by cases: the value of $\operatorname{Var}^{I}\left(\left(a_{0}, \ldots, a_{B}\right), n\right)$ will be $a_{n}$. It is easy to check that so far this interpretation satisfies statements (1)-(4) in Definition 7 and also guarantees that " $B$ exists" is true. 
Rfmla $^{I}$ is likewise defined by cases: it holds precisely for those members of $N^{I}$ which are Gödel numbers of $R$-formulas. The interpretation of $Q$ is very simple; in fact, $Q_{j}^{I}$ can be taken to be $R_{j}$ (for $j \notin J$ there is no harm in assuming that $R_{j}$ is the universally false relation).

The definition of $\operatorname{Sat}_{n}^{I}(f, v)$ comes down to a rather complicated set of cases depending on the values of both $n$ and $f$. Basically, if $f$ is the Gödel number of the $R$-formula $\phi^{f}\left(x_{0}, \ldots, x_{m}\right)$, then $\operatorname{Sat}_{n}^{I}(f, v)$ should hold iff $\psi\left(\operatorname{Var}^{I}(v, 0), \ldots, \operatorname{Var}^{I}(v, m)\right)$ is true, where $\psi$ results from $\phi^{f}$ by replacing $R$ with $R_{n}$. It is left to the reader to check that this interpretation preserves the proper semantics of the satisfaction relation and obeys statements (5) and (6) in Definition 7. A crucial point in the induction is the fact that the arithmetical formulas Ratom, Neg, Disj, and Exist are bounded and therefore absolute.

Finally, the definition of True ${ }^{I}$ is already provided by statement (7) in Definition 7 . Thus $I$ does indeed constitute an interpretation of $\Gamma$. But now it follows from the definition of Sat ${ }^{I}$ that if $\phi^{j}$ is any $R$-sentence with $j \leq B$, then True ${ }^{I}(j)$ holds iff $\bar{\phi}^{j}$ is true. In particular, since $\phi^{J}$ proves $\bar{\phi}^{j}$ for each $j \in J$, the interpretation $I$ makes all of $\Gamma^{J}$ true.

Proof of Lemma 9. Since $\Gamma^{J}$ is logically equivalent to the disjunction of the sentences $\Gamma \wedge$ " $j$ exists" $\wedge \operatorname{True}(j)$ for $j \in J$, Lemma 1 shows that $\left|\Gamma^{J}\right|$ is equal to the meet of the chapters of these sentences. Hence it suffices to prove the lemma just for the case $k=1$, i.e., to show that $\phi^{j}$ is interpretable in $\Gamma \wedge " j$ exists" $\wedge \operatorname{True}(j)$.

The interpretation of $\phi^{j}$ is trivial: the universe is relativized to $M$ and $R$ is interpreted as $Q_{j}$. As in the previous proof, the induction conditions for the Sat relation guarantee that the interpretation works properly.

At last the stage is set for the proof of Theorem 4 . Let $F$ and $I$ be the r.e. indexed collections of sentences mentioned in the theorem. Since they are r.e., there are bounded formulas $F(x, y, z)$ and $I(x, y, z)$ of TA such that

$$
\begin{array}{lll}
b \in F_{a} & \text { iff } & F(\# a, \# b, n) \text { holds for some } n, \text { and } \\
b \in I_{a} & \text { iff } & I(\# a, \# b, n) \text { holds for some } n .
\end{array}
$$

Furthermore, it is possible to choose these formulas so that

$$
\begin{aligned}
& \mathrm{TA} \vdash F(x, y, z) \wedge z \leq z^{\prime} \rightarrow F\left(x, y, z^{\prime}\right) \text { and } \\
& \mathrm{TA} \vdash I(x, y, z) \wedge z \leq z^{\prime} \rightarrow I\left(x, y, z^{\prime}\right) .
\end{aligned}
$$

With this notation in place, the remainder of the proof just consists of writing down appropriate choices for the sentence $\alpha$ and verifying that they are correct.

P art (1). By means of the Fixed-Point Lemma, determine a sentence $\alpha$ with Gödel number $\# \alpha=a$ and a bound $B \geq a$, such that $\alpha$ is provably 
equivalent to

$$
\Gamma \wedge \text { “ } B \text { exists" } \wedge \exists n[F(a, a, n) \wedge \neg I(a, a, n)] .
$$

First, suppose for a contradiction that $\alpha \in F_{\alpha}$. Then there is some $n_{0} \geq B$ satisfying $F\left(a, a, n_{0}\right)$. By assumption $\alpha \notin F_{\alpha} \cap I_{\alpha}$, hence $\alpha \notin I_{\alpha}$, and so $\neg I\left(a, a, n_{0}\right)$ holds. Since $F$ and $I$ are bounded formulas, TA $\wedge$ " $n_{0}$ exists" proves $\exists n[F(a, a, n) \wedge \neg I(a, a, n)]$, so by the limiting case of Lemma $8, \alpha$ has a finite model. But this implies $\alpha \in I_{\alpha}$, which is a contradiction. Thus $\alpha$ cannot be in $F_{\alpha}$.

Now suppose that $\alpha \in I_{\alpha}$. Then there is some $n_{0}$ satisfying $I\left(a, a, n_{0}\right)$, and clearly $\alpha$ implies $\Gamma \wedge$ " $B$ exists" $\wedge \exists n<n_{0} F(a, a, n)$. But $\alpha \notin F_{\alpha} \cap I_{\alpha}$, hence $\alpha \notin F_{\alpha}$, hence $\alpha$ has a provably inconsistent consequence, and hence $\alpha$ is in $F_{\alpha}$ after all, another contradiction. Therefore $\alpha$ cannot be in $I_{\alpha}$ either.

Part (2). As before, find $\alpha$ equivalent to

$\Gamma \wedge$ "B exists" $\wedge \exists n[F(a, a, n) \wedge \neg I(a, a, n)$

$$
\wedge \forall j \leq n(\operatorname{Rfmla}(j) \wedge I(a, j, n) \rightarrow \operatorname{True}(j))] .
$$

By the same reasoning as in part (1), $\alpha \notin I_{\alpha}$.

Now suppose $\alpha \in F_{\alpha}$, and let $n_{0} \geq B$ satisfy $F\left(a, a, n_{0}\right)$. Let

$$
J=\left\{j \leq n_{0} \mid \operatorname{Rfmla}(j) \wedge I\left(a, j, n_{0}\right)\right\}=\left\{j_{1}, \ldots, j_{k}\right\},
$$

and set

$$
\Gamma^{J}=\Gamma \wedge \text { “ } n_{0} \text { exists" } \wedge \operatorname{True}\left(j_{1}\right) \wedge \cdots \wedge \operatorname{True}\left(j_{k}\right) .
$$

Clearly $\Gamma^{J}$ logically implies $\alpha$, so $|\alpha| \leq\left|\Gamma^{J}\right|$. However, by Lemma $8,\left|\Gamma^{J}\right| \leq$ $\left|\phi^{j_{1}}\right| \vee \cdots \vee\left|\phi^{j_{k}}\right|$. Since each $\phi^{j}$ belongs to $I_{\alpha}$ and $I_{\alpha}$ is an ideal, it follows that $\alpha \in I_{\alpha}$, a contradiction. Thus $\alpha \notin F_{\alpha}$.

It is also necessary to show that for any $\phi \in I_{\alpha},|\phi| \leq|\alpha|$. By Lemma 3, there is some $R$-sentence $\phi^{j}$ such that $|\phi|=\left|\phi^{j}\right|$. By Lemma 9, $\left|\phi^{j}\right| \leq$ $\mid \Gamma \wedge$ " $j$ exists" $\wedge \operatorname{True}(j) \mid$, so it suffices to show that $\alpha$ proves " $j$ exists" $\wedge$ $\operatorname{True}(j)$. Since $\phi^{j} \in I_{\alpha}$, there is some $n_{0} \geq j$ satisfying $I\left(a, j, n_{0}\right)$, and since $\alpha \notin F_{\alpha}, F\left(a, a, n_{0}\right)$ is false. It follows that TA $\vdash \forall n\left[F(a, a, n) \rightarrow\left(n \geq n_{0}\right.\right.$ $\wedge I(a, j, n))]$, which yields the desired conclusion.

$\mathrm{P}$ art (3). As before, find $\alpha$ equivalent to

$$
\begin{array}{r}
\Gamma \wedge \text { "B exists" } \wedge \exists n[(F(a, a, n) \vee \exists j \leq n(\operatorname{Rfmla}(j) \wedge F(a, j, n) \wedge \operatorname{True}(j))) \\
\wedge \neg I(a, a, n)] .
\end{array}
$$

By the same argument as in part (1), $\alpha \notin F_{\alpha}$.

Now suppose $\alpha \in I_{\alpha}$, and let $n_{0}$ satisfy $I\left(a, a, n_{0}\right)$. Then $\alpha \notin F_{\alpha}$ so $F(a, a, n)$ is provably false for all $n \leq n_{0}$, while $I(a, a, n)$ is provably true for all $n \geq n_{0}$. Let

$$
J=\left\{j \leq n_{0} \mid \operatorname{Rfmla}(j) \wedge F\left(a, j, n_{0}\right)\right\}=\left\{j_{1}, \ldots, j_{k}\right\} ;
$$


then $\alpha$ implies

$$
\Gamma \wedge\left[\left(" j_{1} \text { exists" } \wedge \operatorname{True}\left(j_{1}\right)\right) \vee \cdots \vee\left(" j_{k} \text { exists" } \wedge \operatorname{True}\left(j_{k}\right)\right)\right] .
$$

Hence by Lemma $9,|\alpha| \geq\left|\phi^{j_{1}}\right| \wedge \cdots \wedge\left|\phi^{j_{k}}\right|$. However, each $\phi^{j}$ belongs to $F_{\alpha}$ and $F_{\alpha}$ is a filter; this implies that $\alpha \in F_{\alpha}$, a contradiction.

To see that $|\phi| \geq|\alpha|$ for every $\phi \in F_{\alpha}$, find an $R$-sentence $\phi^{j}$ such that $|\phi|=\left|\phi^{j}\right|$ as before. There is some $n \geq j$ such that $n \geq B$ and $F(a, j, n)$ holds. By Lemma 8, $\left|\phi^{j}\right| \geq \mid \Gamma \wedge$ " $n$ exists" $\wedge \operatorname{True}(j) \mid$, and since $I(a, a, n)$ is false, this sentence implies $\alpha$.

Part (4). As before, find $\alpha$ equivalent to

$$
\begin{aligned}
& \Gamma \wedge " B \text { exists" } \wedge \exists n {[(F(a, a, n) \vee \exists j \leq n(\operatorname{Rfmla}(j) \wedge F(a, j, n) \wedge \operatorname{True}(j)))} \\
&\wedge \neg I(a, a, n) \wedge \forall j \leq n(\operatorname{Rfmla}(j) \wedge I(a, j, n) \rightarrow \operatorname{True}(j))] .
\end{aligned}
$$

Now the same argument as in part (2) shows that $\alpha \notin F_{\alpha}$, while the same argument as in part (3) shows that $\alpha \notin I_{\alpha}$.

To see that $|\phi| \geq|\alpha|$ for every $\phi \in F_{\alpha}$, find an $R$-sentence $\phi^{j_{0}}$ such that $|\phi|=\left|\phi^{j_{0}}\right|$ as before. There is some $n \geq j_{0}$ such that $n \geq B$ and $F\left(a, j_{0}, n\right)$ holds. Let

$$
J=\left\{j_{0}\right\} \cup\{j \leq n \mid \operatorname{Rfmla}(j) \wedge I(a, j, n)\}=\left\{j_{0}, j_{1}, \ldots, j_{k}\right\}
$$

and let

$$
\Gamma^{J}=\Gamma \wedge \text { " } n \text { exists" } \wedge \operatorname{True}\left(j_{0}\right) \wedge \cdots \wedge \operatorname{True}\left(j_{k}\right) .
$$

Then $\Gamma^{J} \vdash \alpha$, so it suffices to show that $|\phi| \geq\left|\Gamma^{J}\right|$.

By Lemma 8 , in fact it is only necessary to show that $|\phi| \geq\left|\phi^{j_{i}}\right|$ for each $i$. For $i=0$ this is true by the choice of $j_{0}$, and for $i>0$ it is true because $\phi^{j_{i}} \in I_{\alpha}$ and every member of $F_{\alpha}$ can interpret every member of $I_{\alpha}$ (by assumption).

Finally, to see that $|\phi| \leq|\alpha|$ for every $\phi \in I_{\alpha}$, let $\phi^{j_{0}}$ be an $R$-sentence with $|\phi|=\left|\phi^{j_{0}}\right|$ and find $n$ such that $I\left(a, j_{0}, n\right)$ holds. Let

$$
J=\left\{j_{0}\right\} \cup\{j \leq n \mid \operatorname{Rfmla}(j) \wedge F(a, j, n)\}=\left\{j_{0}, j_{1}, \ldots, j_{k}\right\} .
$$

It is then easy to check that $\alpha$ implies the sentence

$$
\Gamma^{J}=\Gamma \wedge\left[\left(" j_{0} \text { exists" } \wedge \operatorname{True}\left(j_{0}\right)\right) \vee \cdots \vee\left(" j_{k} \text { exists" } \wedge \operatorname{True}\left(j_{k}\right)\right)\right] .
$$

Hence by Lemma $9,|\alpha| \geq\left|\phi^{j_{0}}\right| \wedge \cdots \wedge\left|\phi^{j_{k}}\right|$. Since $\phi^{j_{i}} \in F_{\alpha}$ for $i>0$, this meet is equal to $\left|\phi^{j_{0}}\right|$. This yields the desired result, and concludes the proof of Theorem 4.

The three results originally announced in [MPS] are proved below as corollaries of this theorem. But before giving the corollaries it is necessary to explain that the notion of interpretability of theories used in $\mathbf{L C}$ is a local one; that is, a theory $S$ is interpretable in a theory $T$ iff $T$ can interpret 
$\beta$ for every consequence $\beta$ of $S$. Another way of putting it is to say that $|S| \leq|T|$ iff $|\beta| \leq|T|$ for every sentence $\beta$ such that $|\beta| \leq|S|$.

Corollary 10 (Theorem 6.25 of [MPS]). If $S$ and $T$ are r.e. theories such that $|S|<|T|$, then the interval $[|S|,|T|]$ in $\mathbf{L C}$ is not complemented.

Proof. Since $|T| \overleftrightarrow{\leq}|S|$, there is a consequence $\tau$ of $T$ that is not interpretable in $S$. Now for each sentence $\alpha$, let $I_{\alpha}=\{\beta|| \alpha \vee \beta|\leq| S \mid\}$ and $F_{\alpha}=\{\gamma|| \gamma|\geq| \tau \mid\}$. Since interpretability is an r.e. notion, these indexed collections are r.e. Furthermore, using Lemma 1 and the distributivity of LC, $I_{\alpha}$ is an ideal. Since all the other requirements of Theorem 4(2) are also satisfied, there is a sentence $\alpha$ such that $\alpha \notin F_{\alpha}$ and $|\alpha|>|\beta|$ for all $\beta \in I_{\alpha}$.

Let $x=|\alpha| \wedge|T|$. Then it turns out that $|S|<x<|T|$ and $x$ has no lower complement in this interval; i.e., there is no $y$ such that $|S|<y<|T|$ and $x \wedge y=|S|$. To see that this is true, first note that $I_{\alpha}=\{\beta|| \alpha|\wedge| \beta|\leq| S \mid\}$; hence if $|\beta| \leq|S|$ then $\beta \in I_{\alpha}$ and so $|\beta|<|\alpha|$. It follows that $|S| \leq|\alpha|$ and thus $|S| \leq x$. However, $\alpha \notin F_{\alpha}$, so $|\alpha| \nsupseteq|\tau|$, so $\tau \notin I_{\alpha}$, so $|\alpha| \wedge|\tau| \not|S|$, so $|\alpha| \wedge|T| \not \leq|S|$, and thus $|S|<x$. Clearly $x \leq|T|$, and in fact $x<|T|$ because $x \geq|\tau|$. Now suppose there is a lower complement $y$ for $x$. Then $|S| \geq(|\alpha| \wedge|T|) \wedge y=|\alpha| \wedge(|T| \wedge y)=|\alpha| \wedge y$, so for any sentence $\beta$ such that $|\beta| \leq y$, this implies that $\beta \in I_{\alpha}$ and so $|\beta| \leq|\alpha|$. Therefore $y \leq|\alpha|$, so $y \leq x$ and $|S|=x \wedge y=y$, a contradiction.

In the case that $S$ and $T$ are finitely axiomatizable, it is possible to find a sentence $\alpha$ such that $|S|<|\alpha|<|T|$ and $|\alpha|$ has neither an upper nor a lower complement in this interval. Simply use part (4) of Theorem 4, with

$$
\begin{aligned}
I_{\alpha} & =\{\beta|| \beta|\leq| T \mid \text { and }|\alpha| \wedge|\beta| \leq|S|\} \quad \text { and } \\
F_{\alpha} & =\{\gamma|| \gamma|\geq| S \mid \text { and }|\alpha| \vee|\gamma| \geq|T|\} .
\end{aligned}
$$

It is necessary to know that $S$ and $T$ are finitely axiomatizable for $F_{\alpha}$ to be r.e., because this means for example that $|\gamma| \geq|S|$ iff $\sigma$ is interpretable in $\gamma$, where $\sigma$ is an axiom for $S$. It is easy to check that $I_{\alpha}$ is an ideal, $F_{\alpha}$ is a filter, and $\alpha \notin I_{\alpha} \cup F_{\alpha}$. Lastly, whenever $\beta \in I_{\alpha}$ and $\gamma \in F_{\alpha}$ it is true that

$$
\begin{aligned}
|\beta| & =|\beta| \wedge|T| \leq|\beta| \wedge(|\alpha| \vee|\gamma|) \\
& =(|\beta| \wedge|\alpha|) \vee(|\beta| \wedge|\gamma|) \leq|S| \vee|\gamma|=|\gamma| .
\end{aligned}
$$

By Theorem 4, there is a sentence $\alpha$ such that $|\beta|<|\alpha|<|\gamma|$ for all $\beta \in I_{\alpha}$, $\gamma \in F_{\alpha}$. This implies that $|S|<|\alpha|<|T|$. The same sort of argument as above shows that $|\alpha|$ has no lower complement. If $y$ is an upper complement for $|\alpha|$, then by compactness there is a sentence $\gamma$ such that $|S| \leq|\gamma| \leq y$ and $|\alpha| \vee|\gamma|=|T|$, which implies that $\gamma \in F_{\alpha}$ and hence $|\alpha|<|\gamma|$. This means that $|\gamma|=|\alpha| \vee|\gamma|=|T|$, so $y \geq|T|$ and therefore is not an upper complement after all. 
CoROLlaRY 11 (Theorem 6.26 of [MPS]). If $S$ is an r.e. theory and $\tau$ is a sentence not interpretable in $S$, then there is a sentence $\alpha$ such that $|S|<|\alpha|$ and $|\tau| \not \leq|\alpha|$. Additionally, if $|S|<|\tau|$ then $\alpha$ can be chosen so that $|S|<|\alpha|<|\tau|$.

Proof. Use part (2) of Theorem 4, with $I_{\alpha}=\{\beta|| \beta|\leq| S \mid\}$ and $F_{\alpha}=\{\gamma|| \tau|\leq| \gamma \mid\}$. If $|S|<|\tau|$, use part (4) of Theorem 4 with the same $I_{\alpha}$ and $F_{\alpha}$.

This corollary is not very interesting in itself, but it immediately implies that for every r.e. theory $S,|S|$ is the meet of $\{|\alpha||| S|<| \alpha \mid\}$. Indeed, if $|S|$ were not equal to this meet there would have to be a sentence $\tau$ such that $|\tau| \leq|S|$ and $|\tau| \leq|\alpha|$ for every $|\alpha|>|S|$, contradicting the corollary. Another interesting consequence concerns versions of set theory. GB set theory is finitely axiomatizable, and ZF set theory is r.e. and interpretable in GB but not vice versa. By the second part of the corollary, there is a finitely axiomatizable theory $T$ strictly intermediate between the two: $|\mathrm{ZF}|<|T|<|\mathrm{GB}|$.

Corollary 12 (Theorem 6.28 of [MPS]). Let $S$ be an r.e. theory and let $A$ be an r.e. collection of sentences such that for any finite $A_{0} \subseteq A$, $|S|<\bigwedge\left\{|\tau| \mid \tau \in A_{0}\right\}$. Then there is a sentence $\alpha$ such that $|S|<|\alpha| \leq$ $\bigwedge\{|\tau| \mid \tau \in A\}$.

Proof. Use part (4) of Theorem 4, with $I_{\alpha}=\{\beta|| \beta|\leq| S \mid\}$ and $F_{\alpha}=\left\{\gamma \mid \exists \tau_{1}, \ldots, \tau_{n} \in A\left(\left|\tau_{1} \vee \cdots \vee \tau_{n}\right| \leq|\gamma|\right)\right\}$. Then $I_{\alpha}$ and $F_{\alpha}$ are r.e., and by Lemma $1, F_{\alpha}$ is a filter (in fact, it is the filter generated by $A)$. For any $\beta \in I_{\alpha}$ and $\gamma \in F_{\alpha}$ there are $\tau_{1}, \ldots, \tau_{n} \in A$ such that $|\beta| \leq$ $|S|<\left|\tau_{1}\right| \wedge \cdots \wedge\left|\tau_{n}\right| \leq|\gamma|$. This shows the requirements of Theorem 4 are satisfied, and so there is a sentence $\alpha$ such that $|S| \leq|\alpha|$ and $|\alpha|<|\tau|$ for every $\tau \in A$. But $|S| \neq|\alpha|$ because otherwise $\alpha$ would be in $I_{\alpha}$, so $|S|<|\alpha| \leq \bigwedge\{|\tau| \mid \tau \in A\}$

As a consequence of this corollary, if $A$ is an r.e. collection of sentences none of which has a finite model, then there is a sentence $\alpha$ which also has no finite models but is interpretable in every member of $A$.

\section{References}

[K] J. Krajíčck, Some theorems on the lattice of local interpretability types, Z. Math. Logik Grundlag. Math. 31 (1985), 449-460.

[MPS] J. Mycielski, P. Pudlák and A. Stern, A lattice of chapters of mathematics (interpretations between theorems), Mem. Amer. Math. Soc. 426 (1990).

[T] B. A. Trachtenbrot, On recursive inseparability, Dokl. Akad. Nauk SSSR 88 (1953), 953-956 (in Russian). 
[V] R. L. Vaught, Axiomatizability by a schema, J. Symbolic Logic 32 (1967), 473479 .

THE ROWLAND INSTITUTE FOR SCIENCE

100 CAMBRIDGE PARKWAY

CAMBRIDGE, MASSACHUSETTS 02142

U.S.A.

Received 8 August 1992 